\title{
A Study on Performance and Cavitation of Propellers for High Speed Crafts Including Effect of Boss
}

\author{
(2nd Report: Analysis in Oblique Flow)
}

\author{
by Sudeb Chattopadhyay*, Member Hiroharu Kato**, Member \\ Hajime Yamaguchi**, Member
}

\begin{abstract}
Summary
In the first report the authors discussed about the analysis in uniform flow. Using the same discrete model, the analysis for the present report was performed for the oblique case. In addition to the effect of boss, propeller slipstream asymmetry was also incorporated into the model. Experiments were performed by inclining the propeller shaft with respect to the flow direction in the cavitation tunnel. Predicted cavity extents had been found to agree relatively well with the observed ones when the predicted values were advanced by sixty degrees. In the calculation of pressure distribution on the blade, it had been found that there was a considerable drop in pressure at about $60 \%$ chord position of the blade near the boss and this position had been found to agree very well with the observed position of root cavitation erosion.
\end{abstract}

\section{Introduction}

In case of high speed crafts, because of speed requirements, it is of ten necessary that the propeller be mounted on an inclined shaft to accommodate the minimum required diameter. These crafts because of their typical shape of ten has no significant hull wake. Thus the variation of inflow to the propeller disc mainly arises out of the inclination of the shaft. When the flow is inclined, it gives rise to tangential and radial components of wake varying with a fundamental once-per-revolution frequency. This is in contrast to the single screw merchant ship wake, where there is principally once-per-revolution variation of longitudinal wake, the tangential or radial wake being comparably negligible. In view of this care must be taken about the three facts, namely, the variation in tangential wake, the asymmetry of the propeller slipstream and the presence of the boss as a bound. ary near the root region where the variation of angle of attack is maximum.

As a first step to study this problem the authors

* Graduate Student, Department of Naval Architecture, University of Tokyo at the time of the study ; Presently, Research Engineer, Ship and Offshore Division, Ishikawajima-Harima Heavy Industries Co. Ltd., Tokyo

** Department of Naval Architecture, University of Tokyo reported $^{1)}$ on the analysis in uniform flow in which the effect of boss was also considered in the VLM (Vortex Lattice Method) on which the whole study ${ }^{2)}$ was based.

Very few studies related to propellers in oblique flow ${ }^{3) \sim 8}$ had been published so far. Among them lifting surface theory was applied by Kerwin ${ }^{5)}$ and Boswell ${ }^{6}$. Kerwin had taken into account the effect of propeller slipstream asymmetry in his VLM model and Boswell reported on the application of this model to predict periodic single blade loads on propellers in tangential wake. In this study, in addition to the slipstream asymmetry, the effect of boss on pressure distribution on the blade, specially near the root region had been studied. The simulation results had been assessed based on the experimentally observed cavitation extents and erosion pattern.

\section{Formulation of Numerical Lifting Surface Theory}

The details of the basic theory adopted in this study had been reported in ${ }^{1), 2)}$. However, for ease of reference the part of the model relevant to oblique flow analysis will be briefly described here. For the details of blade and boss singularity distribution the reader is referred to ${ }^{1)}$.

2. 1 Basic assumptions regarding flow conditions

The propeller blades are considered to be a set of symmetrically arranged thin blades fo arbitrary 
form rotating with a constant angular velocity about a common axis in an unbounded fluid. For the present analysis, the presence of any extraneous solid boundaries except the propeller hub is ignored.

The blades are assumed to be sufficiently thin and operate at small angles of attack so that their presence in the fluid can be represented by the distribution of vortices and sources lying on the mean camber surface of each blade, together with a distribution of vortices shed into the slipstream wake.

When the shaft is inclined at a certain angle to the inflow direction a velocity component tangential to the direction of propeller rotation is generated. The tangential wake thus generated depends upon the main inflow and the corresponding shaft inclination angle. Thus the magnitude of maximum tangential wake is constant throughout from tip to the boss. But since the tangential velocity due to blade rotation is smaller at the boss compared to the tip, the variation of angle of attack is larger at the root than at the tip. Therefore in formulating the lifting surface theory for application to oblique flow, care must be taken to adequately consider the change of tangential inflow in one revolution. If one takes into account a constant downward velocity component along with the axial inflow, the oblique flow effects can be adequately simulated. In the present program the input wake parameters are required for the $x, y, z$ (see Fig. 1 in $^{1)}$ ) directions respectively and the oblique flow is simulated by considering a constant velocity $\left(V_{A} \sin \alpha^{\prime}\right)$ in the $y$ direction along with the suitable axial inflow, the $z$ component being set to zero at the propeller plane, where $V_{A}$ is the advance velocity and $\alpha^{\prime}$ is the shaft inclination.

\subsection{Discretization of trailing vortex wake singularities}

The presence of the trailing vortex wake significantly influences the accuracy of the calculation of the induced velocities on the blade. The details of the singularity arrangement used in this analysis was similar to that described in ${ }^{1)}$, with the exception that slipstream asymmetry was taken into account.

When the inflow to the propeller is inclined with respect to the propeller axis, the propeller slipstream is coaxial with the propeller shaft immediately downstream of the blade but further downstream it gradually becomes coaxial with the direction of the main inflow, thus leading to the asymmetry of the propeller slipstream with respect to the propeller shaft axis. Fig. 1 illustrates the asymmetric propeller slipstream singularity arrangement adopted in this study. For simplicity in calculation, the axis of the slipstream is taken as a straight line and the point of deviation from

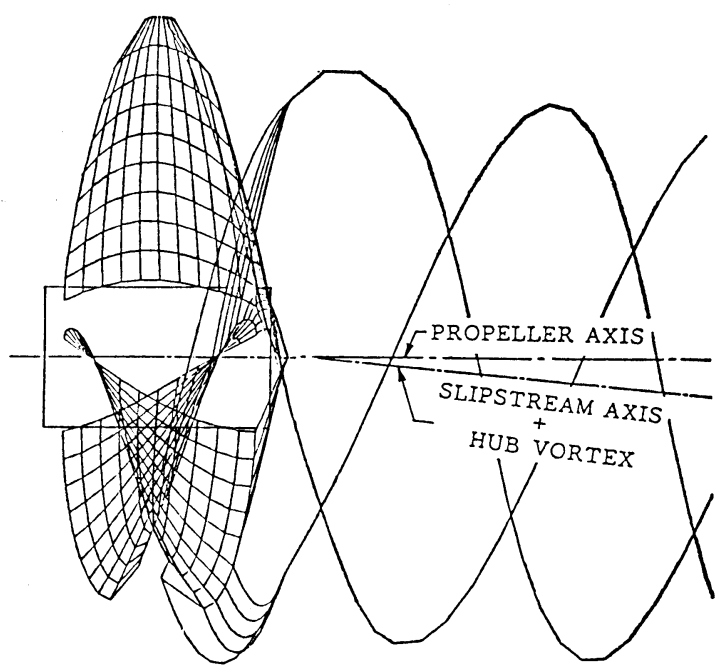

Fig. 1 Propeller slipstream singularity arrangement for asymmetric wake

the propeller axis is taken about $5 \%$ downstream of the axial blade trailing edge position ( $x$-coordinate) at midspan of the blade. From the tip axial position to this point of deflection the slipstream is assumed to be coaxial with the propeller shaft.

In the axisymmetric case, the relative position of the wake singularities with respect to a control point on the blade remains the same as the blade rotates through $360^{\circ}$ along with the wake trailing behind it. Thus the velocity induced by a vortex element of unit strength in the slipstream (or wake) remains constant throughout one revolution and is a function of the relative position of the blade control point and the shed vortex singularity. In contrast, in the asymmetric case the relative position of a wake vortex element with respect to a certain control point on the blade changes in a once-per-revolution cycle. Thus the influence function no longer remains constant throughout one revolution and instead becomes a function of the angular coordinate $\theta$ and the relative distance between the two points in question. If a time-saving iterative computation is envisaged in such a case the storage requirements for the influence function of the wake singularity is required to be increased as many times the number of time steps in one revolution. This calls for enormous memory requirements, since the number of time steps in one revolution is 60 which is generally thought to be the minimum required to study the variation in the first order harmonic of the unsteady loadings on propeller blade. On the other hand, if instead of storing the value of the influence function at each angular position, it is computed at every time step, then 
the time required for the iterative calculation is enormous, if not prohibitive, since the calculations have to be performed for about 240 time steps, i. e. about 4 complete revolutions of the blade in order to obtain proper convergence. Thus an approximate computation procedure to treat the propeller slipstream asymmetry, without much sacrifice in the ultimate accuracy and yet quite fast has been developed. Investigating by direct computation the variation of the influence function of a shed vortex quadrilateral at a particular tangency control point on the key blade, one will realize that it can be approximated by a cosine function of the angular coordinate. To approximate such influence functions it is required that three values for each shed vortex quadrilateral, namely, the mean, the amplitude and the phase with respect to the generator line be estimated. Thus the calculation can be carried out with only an increase of the memory twice that of the number of shed vortex quadrilaterals in the axisymmetric case. In the actual calculation the influence function for the shed vortex quadrilaterals are calculated at a small number of steps (the angular interval being 3 or 4 times that of each time step advance) and then the mean, amplitude and phase ascertained from these values. Such an approximation results in considerable saving in computation time.

The position of the shed vortices are obtained by joining the corresponding ends of the trailing vortices. In this case the time step should be such that $d_{\theta}=w \cdot d t$, where $w$ is the angular velocity. The amount of shed vorticity is obtained from the principle of conservation of vorticity at respective chordwise panel, and the sequence of shedding is set such that the $n$-th shed vortex of the present time step is equal to the $(n-1)-$ th shed vortex of the preceding time step. In such a scheme, at a particular time step $N_{t}$ shed vortices upstream of roll up point are assumed to be present, since beyond the roll up points the shed vorticity cannot be mathematically conceived. In a real fluid, it is most likely that the shed vorticity pattern becomes highly disorganized after a short distance behind the trailing edge and hence it can be said that not much error is incurred by ignoring the effects of shed vorticity beyond the roll up points ${ }^{9)}$.

\section{3 Boundary conditions and related equa-} tions

To solve for the unknown strengths of the blade vortices and boss sources, the boundary condition imposed is that the flow must be tangent to the blade as well as boss surface.

In the unsteady case (oblique flow in the present analysis), compared to the steady case, one has to take into account the shed vortices also. Therefore starting flow tangency equation will take the form

$$
\begin{aligned}
0= & \sum_{m=1}^{M}\left(\sum_{n=1}^{N} F_{i n m} s \cdot G_{n m} s+\sum_{n=1}^{N_{t}} F_{i n m} w \cdot G_{n m} w\right) \\
& +\sum_{m=1}^{M_{b}} \sum_{n=1}^{N_{b}} H_{i n m}{ }^{b} \cdot B_{m n}+\sum_{m=1}^{M+1}\left[\sum_{n=1}^{N} F_{i n m}{ }^{c} \cdot G_{n m}{ }^{c}\right. \\
& \left.+\sum_{n=1}^{N_{t}-1} F_{i n m}{ }^{t} \cdot G_{m n}{ }^{t}\right]+n_{i}\left(V_{i} I+V_{i} Q+V_{i} O\right)
\end{aligned}
$$

for $i=1,2 \cdots \cdots M_{X} N+M_{b X} N_{b}$ control points, where the term containing $G_{m n} w$ represents the contribution due to the shed vortices and is the extra term compared to the similar equation for the steady case (equation 20 in (1)). In equation (1) the superscripts $(s, c, t, w)$ stand for spanwise, chordwise, trailing and shed vortices, $F_{i n m}$ and $H_{i n m}$ stand for the influence functions of the $n m-$ th vortex and source singularity respectively at the $i$-th control point, $V_{i} I, \quad V_{i} Q, V_{i} O$ denote the inflow velocity induced by the singularities on the other blades and slipstream respectively, $M_{b}$ and $N_{b}$ are the number of circumferencial and longitudinal divisions on boss between two adjacent blades, $M$ and $N$ are the number of spanwise and chordwise divisions on the blade and $N_{t}$ denotes the number of shed vortices upto roll up point.

From Kelvin's theorem of conservation of vorticity around a chordwise panel one obtains,

$$
G_{l m} w=-\sum_{n=1}^{N} G_{n m} s-\sum_{n=2}^{N_{t}} G_{n m} w
$$

Since the strengths of the trailing vortex is the same as the last chordwise vortex, one can write,

$$
G_{m} t=\sum_{l=1}^{N}\left(G_{l, m-1}{ }^{s}-G_{l, m} s\right)
$$

Substituting this equation and equation (2) in equation (1) and rearranging the summation of the shed vortices, one can write

$$
\begin{aligned}
\sum_{m=1}^{M} \sum_{n=1}^{N} & \left\{F_{\imath n m} s+\sum_{l=n}^{N}\left(F_{\imath \imath(m+1)}{ }^{c}-F_{\imath \imath m^{c}}\right)+F_{\imath \imath m} w\right\} G_{n m} s \\
& +\sum_{n=2}^{N_{t}}\left\{F_{\imath(n-1) m^{w}}+F_{\imath(n-1)(m+1)^{t}-F_{\imath(n-1) m}}\right. \\
& \left.-F_{\imath n m} w\right\} T_{m}{ }^{(t-n+1)}+\sum_{m=1}^{M_{b}} \sum_{n=1}^{N_{b}} H_{\imath n m^{b}} \cdot B_{n m} \\
& =-\left(V_{\imath} I+V_{\imath} Q+V_{\imath} O\right)
\end{aligned}
$$

where $T_{m}{ }^{(t)}$ is the total circulation around the $m$-th chordwise panel at a time $t$,

$$
T_{m}{ }^{(t)}=\sum_{n=1}^{N} G_{n m} s(t)
$$

$t$ being the index of time for the present time step. As can be seen the coefficient of $G_{n m}$ sepresents the induced velocity due to a closed horseshoe vortex made up of the $(n, m)$-th spanwise vortex on the blade, the first shed vortex and the two chordwise vortices joining the ends of 
the spanwise vortices with that of the first shed vortex in the slipstream. Similarly the coefficient of the total circulation $T_{m}{ }^{(t-n+1)}$ represents the velocity induced by the shed vortex quadrilaterals formed by joining the ends of the two consecutive shed vortices with the corresponding trailing vortices.

\subsection{Solution for vortex(blade) and source (boss) strengths}

Before solving equation (4), the strengths of the bound vortices and the boss line sources are obtained for the steady case using the mean of the unsteady axial velocity. Before the unsteady or non-uniform wake is turned on, these steady values of strength of bound vortices and boss sources are assigned to each blade and the part of the boss associated with each blade. The blade is then rotated through a small angular interval of $d_{\theta}$. The strengths of bound vortices and the strengths of the boss sources at this position is taken to be unknown only for the key blade and simultaneous equations given by equation (4) are formulated at control points similar to that in the steady case (1). For the first time step the strength of previous shed vortices are all taken to be zero. As the blade advances from one step to the next, the strength of the vortex that is shed into the wake is determined by equation (2). Thus the strengths of the first shed vortex for the present time step is taken as the strength of second shed vortex for the next time step. These values of bound vortices, boss sources and shed vortices for each time step are stored for updating the values for the next blade as it enters into a position previously occupied by the key blade and thus the strength of bound vortices, boss sources being known. This iteration process is repeated for three or four revolutions of the blade till the solution converges to a steady pattern.

\subsection{Calculation of thrust, torque and pres- sure distribution}

The only difference in this case compared to the steady case is the existence of a force proportional to the time rate of change of the velocity potential, which follows from the unsteady term in the Bernoulli's equation

$$
F_{u \imath j}=\rho(d / d t)\left(\phi^{+}-\phi^{-}\right) d l
$$

where $\left(\phi^{+}-\phi^{-}\right)$denote the jump in potential across the camber surface, and $F_{u i j}$ denotes the force acting on unit spanwise length.

In the present dicretized case equation (6) can be written as

$$
F_{u i j}=\rho n_{n m} \cdot(d / d t)\left(\sum_{k=1}^{n} G_{k m} s\right) \Delta l_{i j}
$$

where $n_{n m}$ denotes the unit normal vector to the blade camber surface at the midpoint of the $(n, m)-$ th spanwise vortex. The time derivative in the equation (7) was obtained by five-point numerical differenciation of the discrete vortex strengths obtained at previous discrete time steps.

Thus the thrust $T(\theta)$ and torque $Q(\theta)$ at a particular angular position given by $\theta$ is calculated from the following relation

$$
\begin{aligned}
T(\theta)= & -\sum_{m=1}^{M} \sum_{n=1}^{N}\left(F_{n m} x^{s}+F_{n m} x^{c}+F_{u n m} x^{s}\right) \\
& -\int_{r_{H}}^{R} F_{D} \sin \left(A^{\prime}\right) d r \\
Q(\theta)= & \sum_{m=1}^{M} \sum_{n=1}^{N}\left\{\left(F_{n m} z^{s}+F_{u n m} z^{s}\right) \cos \left(\theta_{n m} s\right)\right. \\
& -\left(F_{n m} y^{s}+F_{u n m} y^{s}\right) \sin \left(\theta_{n m}{ }^{s}\right)-F_{n m} y^{c} . \\
& \left.\sin \left(\theta_{n m}{ }^{c}\right)+F_{n m} z^{c} \cdot \cos \left(\theta_{n m}^{c}\right)\right\} r_{n m} \\
& +\int_{r_{H}}^{R} F_{D} \cdot \sin \left(A^{\prime}\right) r d r
\end{aligned}
$$

where $F_{u n m} x, \quad F_{u n m} y, \quad F_{u n m} z$ represent the $x, y, z$ components of the force due to the time derivative respectively.

The pressure is computed at the spanwise load points, using the total velocity computed at these points required for force computations. While calculating the pressure coefficient from the Bernoulli's equation, the unsteady term is also taken into account.

\section{Discussion of Computer Simulation and Experimental Results}

A computer program had been developed based on the above mentioned theory. The calculations were performed for three cases, respectively considering propeller slipstream axisymmetry without any boss effects, propeller slipstream axisymmetry with boss effects and propeller slipstream asymmetry without any boss effects. The effect of both the boss and slipstream asymmetry could not be studied together because of storage capacity problems with the computer, but from these results it can be said that if this could be done this would further improve the usefulness of the present method in predicting root cavitation erosion. Experiments were also performed to assess the validity of the calculated results. The principal particulars of the propeller used in this study is shown in Table 1 .

Table 1 Principal particulars of model propeller

\begin{tabular}{lr}
\hline Diameter (mm) & 200 \\
No. of blades & 3 \\
Expanded area ratio & 1.085 \\
Pitch ratio at $0.7 \mathrm{R}$ & 1.125 \\
Section type & Circular back \\
Boss ratio & 0.28 \\
\hline
\end{tabular}

\section{1 Experiment procedure}

To perform experiments in oblique flow it is 


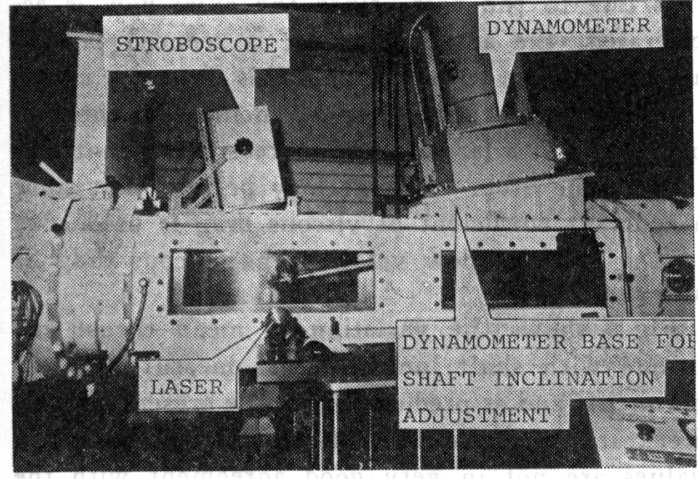

Fig. 2 Experimental set-up

required that the dynamometer be placed on an inclined base so that the propeller shaft can be placed at the desired angle with respect to the main flow direction in the test section. It was found that a shaft inclination more than $10^{\circ}$ was not possible in the cavitation tunnel. Hence the experiments were performed at $5^{\circ}$ and $10^{\circ}$ shaft inclinations, and one base for each of these inclinations was constructed.

An experimental set up just opposite to the actual ship case was used to avoid any unwanted effects due to the dynamometer boss on the inflow to the propeller. This resulted in a reverse inclination and consequently reverese tangential inflow to the propeller as compared to the ship case. The experimental setup adopted in this study is shown in Fig. 2. Since the aim of the present
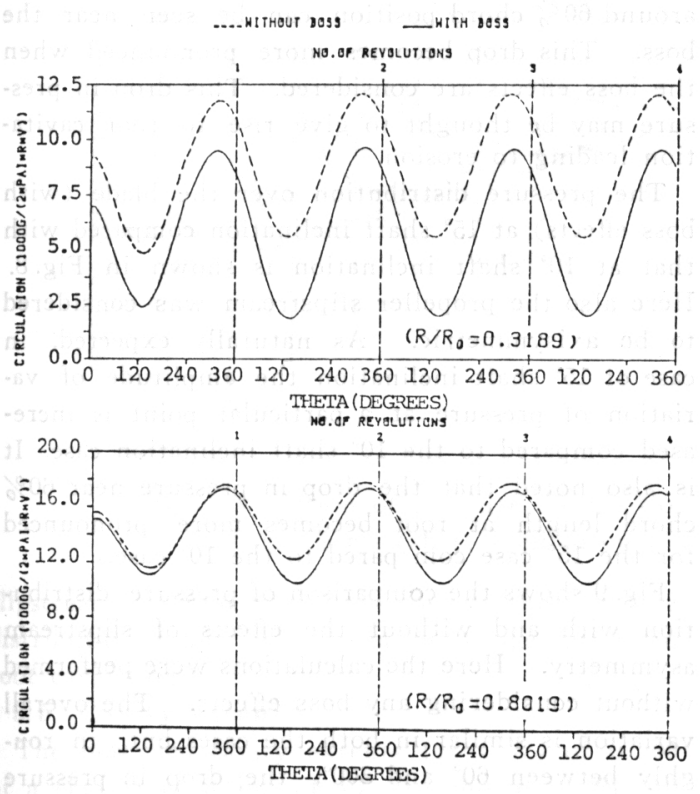

Fig. 3 Convergence of total circulation on blade with and without boss effects ( shaft inclination $=10^{\circ}$ ) study was to investigate the effect of oblique flows, the configuration chosen was sufficient for the purpose.

\section{2 Circulation distribution}

The comparison of the unsteady circulation with and without boss effects are shown in Fig. 3 at two radial positions one near the boss and another near the tip. The slipstream was assumed to be axisymmetric here. Here it can be seen that because of acceleration of the axial inflow due to the presence of the boss the overall circulation is reduced. This reduction is more pronounced near the boss and gradually diminishes at the tip. This is due to the fact that the acceleration of the flow is larger towards the boss than at the tip. In this figure the circulation given by $G$ has been normalized by $R$ (the local radius) and $V$ (the nominal inflow velocity at $0.7 \times R_{0}$.

\subsection{Thrust and torque}

Fig. 4 shows the variation of thrust and torque per blade with and without effects of boss, the slipstream being assumed to be axisymmetric. The calculations had been performed for a shaft inclination of ten degrees. In this figure the thrust and torque show once-per-revolution variation.

Fig. 5 shows the variation of thrust and torque for all the blades, with and without boss effects. Here both thrust and torque show a 3-cycle-perrevolution variation which is equal to the number of blades. For the results considering boss effects, near about $120^{\circ}$ and $240^{\circ}$ some unrealistic patterns are observed. This is the position where each subsequent blade data is interchanged as it enters
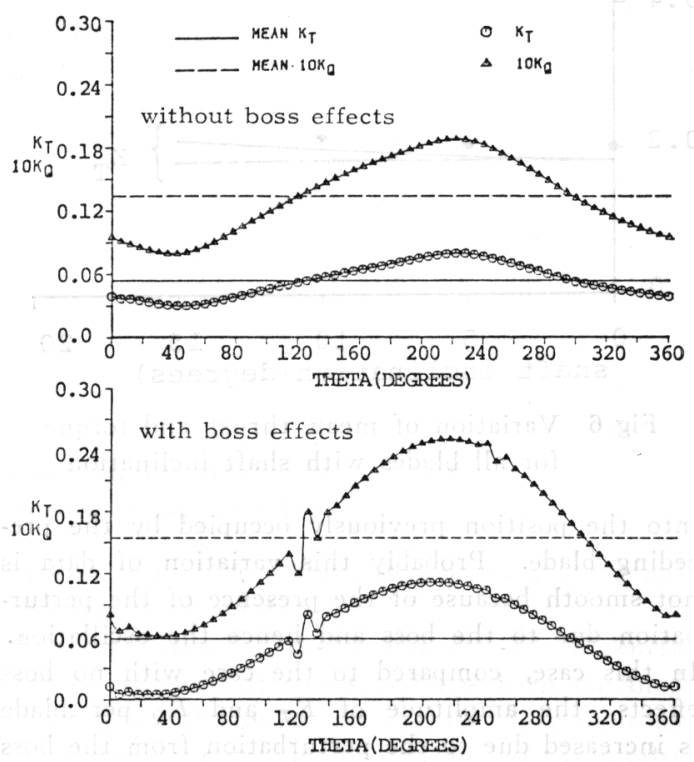

Fig. 4 Variation of $K_{T}$ and $K_{Q}$ per blade with and without boss effects (shaft inclination $=10^{\circ}$ ) 


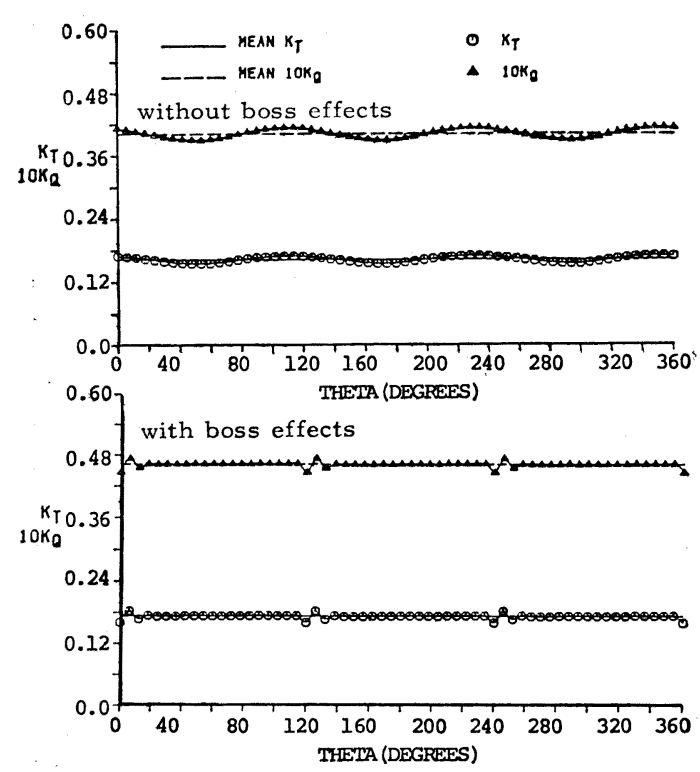

Fig. 5 Variation of $K_{T}$ and $K_{Q}$ for all blades with and without boss effects (shaft inclination $=10^{\circ}$ )

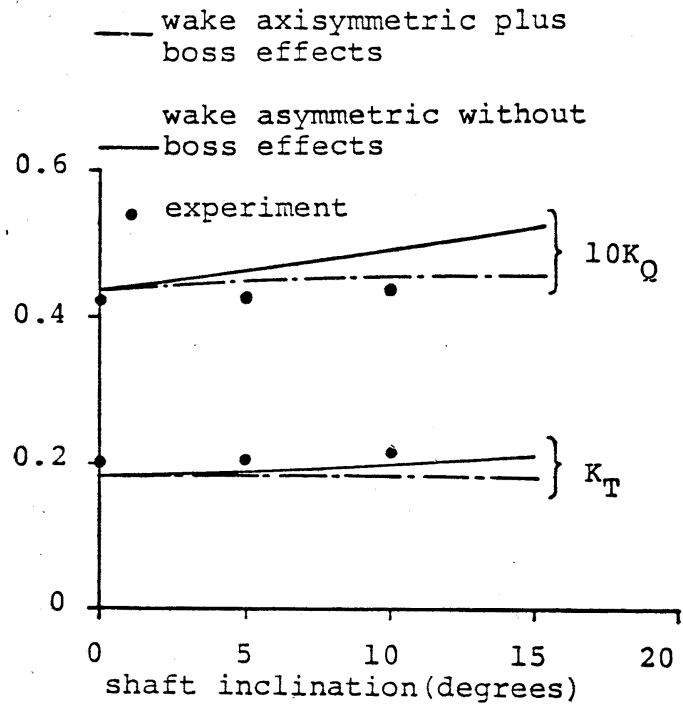

Fig. 6 Variation of mean thrust and torque for all blades with shaft inclination

into the position previously occupied by the preceding blade. Probably this variation of data is not smooth because of the presence of the perturbation due to the boss and hence the oscillation. In this case, compared to the case with no boss effects, the amplitude of $K_{T}$ and $K_{Q}$ per blade is increased due to the perturbation from the boss sources and although there is no appreciable change in the overall $K_{T}$ value, the $K_{Q}$ value is slightly increased. This can be explained from the fact that due to the boss, since the axial flow is accelerated, the angle of attack is reduced causing a reduction in circulation. This in turn causes the lift force generated to be inclined to the propeller axis at a larger angle increasing its contribution to the drag. Also it may be said that since a constant coefficient of viscous drag is used, due to the larger inflow velocity the viscous drag is also increased.

Fig. 6 shows the variation of mean thrust and torque with shaft inclination as measured in experiments and as predicted from the present theory. Here it can be seen that though the predicted values are not in very good agreement with the experimental ones, if the slipstream asymmetry is considered the trend in variation as observed in experiments is simulated by the present theory.

\subsection{Pressure distribution}

The pressure distribution at different radial position with and without boss effects are next compared and the results are shown in Figs 7. The calculations were performed considering the slipstream to be axisymmetric. As can be seen from these figures the amplitude of variation of pressure at a particular point on the blade is slightly increased when the boss effects are considered. As the blade moves from $30^{\circ}$ to $270^{\circ}$, the pressure profile is quite similar to that observed under uniform flow conditions, the pressure being low near the leading edge and higher near the trailing edge. But as the blade next moves from $270^{\circ}$ to $30^{\circ}$, the situation reverses and pressure profiles with higher values near the leading edge and lower values at around $60 \%$ chord position can be seen near the boss. This drop becomes more pronounced when the boss effects are considered. This drop in pressure may be thought to give rise to root cavitation leading to erosion.

The pressure distribution over the blade (with boss effects) at $15^{\circ}$ shaft inclination compared with that at $10^{\circ}$ shaft inclination is shown in Fig. 8. Here also the propeller slipstream was considered to be axisymmetric. As naturally expected, in case of $15^{\circ}$ shaft inclination the amplitude of variation of pressure at a particular point is increased compared to the $10^{\circ}$ shaft inclination case. It is also noted that the drop in pressure near 60\% chord length at root becomes more pronounced for the $15^{\circ}$ case com pared to the $10^{\circ}$ case.

Fig. 9 shows the comparison of pressure distribution with and without the effects of slipstream asymmetry. Here the calculations were performed without considering any boss effects. The overall variation is similar in both the cases but, in roughly between $60^{\circ}$ and $300^{\circ}$, the drop in pressure at about $60 \%$ chord length near the root region is much more pronounced in the slipstream asymmetric case. Therefore, it can be said that if the 


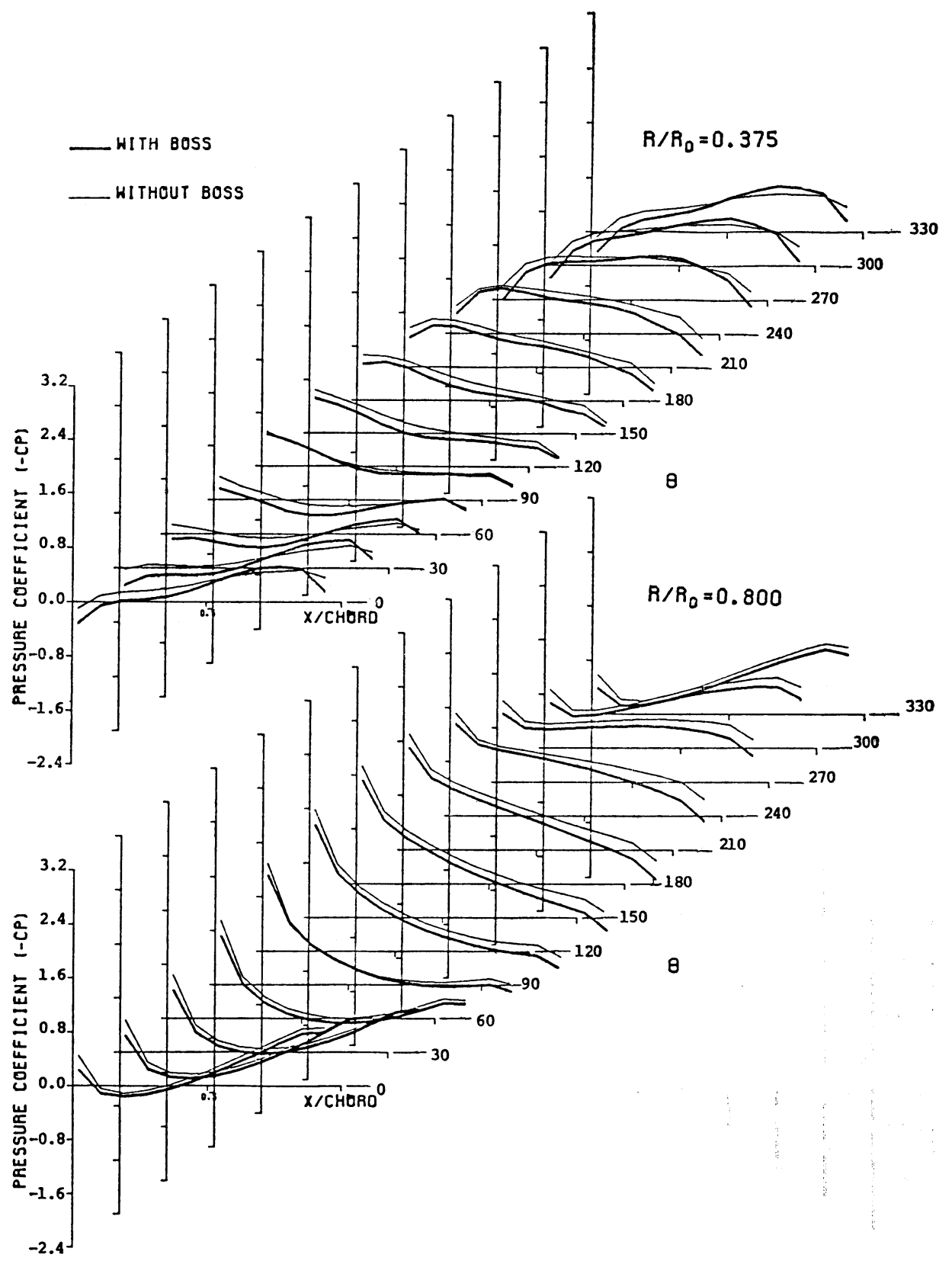

Fig. 7 Variation of pressure distribution on blade (suction side) with and without boss effects (shaft inclination $=10^{\circ}$, slipstream axisymmetric)

slipstream asymmetry is considered, the pressure distribution takes up a form more susceptible to root cavitation.

\subsection{Cavity extent}

The observed cavity extents for a $K_{T}$ of 0.205 at a shaft inclination of $10^{\circ}$ are shown in Fig. 10 for three cavitation numbers. In this case there is no face cavitation, but bubble appear on the back at around $240^{\circ}$ leading to formation of cloud cavity at around $0^{\circ}$. This cloud cavitation is generated only at $\sigma_{n}=0.8$.

The estimation of cavity extent and thickness based on vortex lattice method has been outlined by Lee (10). A similar but slightly more rigorous version was attempted by Yuasa (11), but the results obtained were not very promising. It was therefore decided to ascertain how the most widely used lift-equivalent method results were for this 


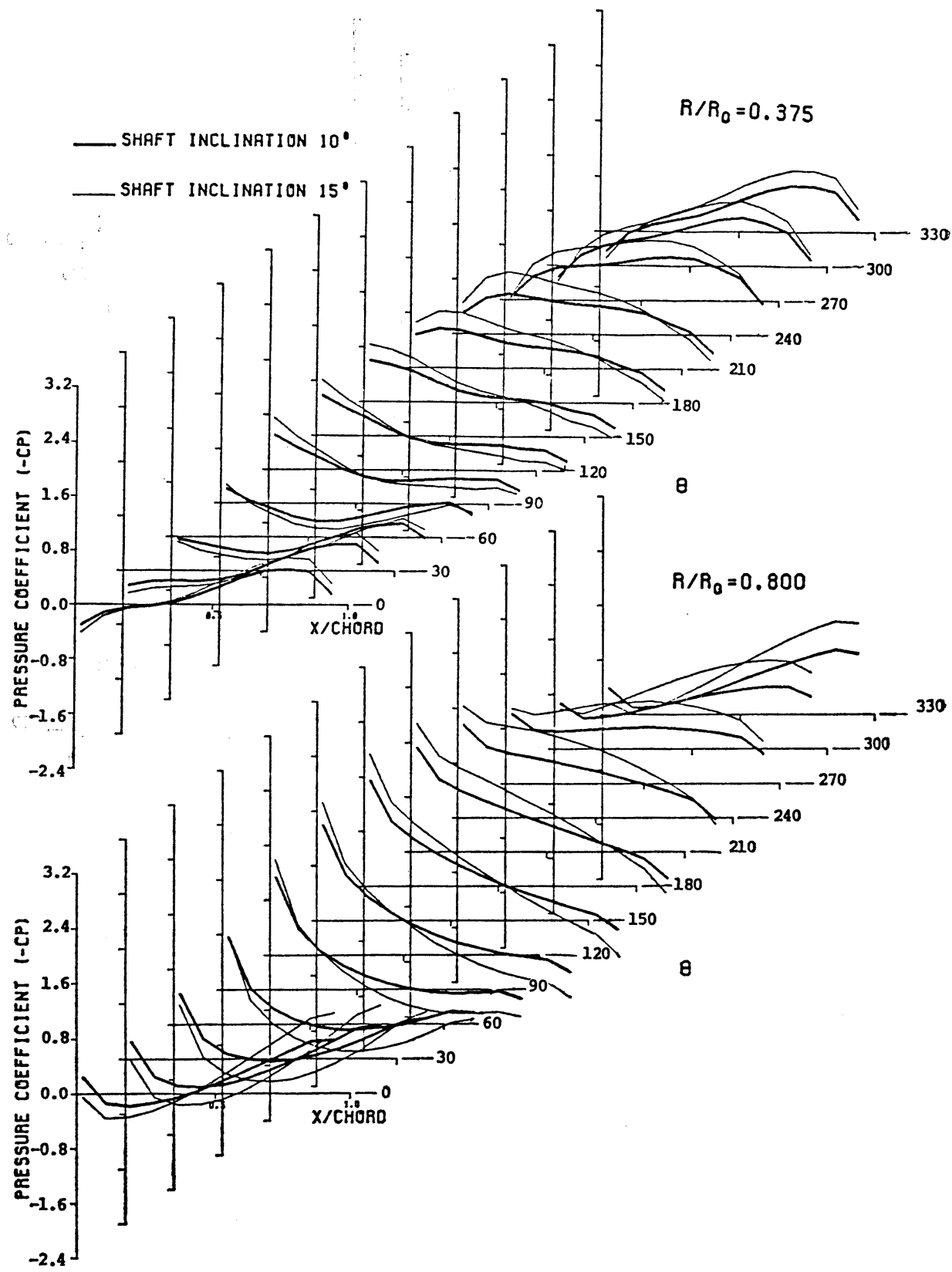

Fig. 8 Variation of pressure distribution on blade (suction side) with shaft inclination (including boss effects, slipstream axisymmetric)

case with such low aspect ratio propeller. Since in the lift-equivalent method no unsteady effects of cavity growth can be taken into account the calculated cavity extents do not agree well with experimental results if a direct comparison is made. However, for the present propeller, if the predicted extent is advanced through about $60^{\circ}$, the results seem to match each other quite closely. This is illustrated in figures 11 and 12, for a cavitation number equal to 0.8 . In Fig. 11 the results considering wake axisymmetry with and without boss effects are shown. It can be seen that the results without boss effects are closer to the experimental results. Fig. 12 shows the results for the wake asymmetric case without any boss effects. Here it is found that the results for the asymmetric case are closer to the experimental results compared to the results. 


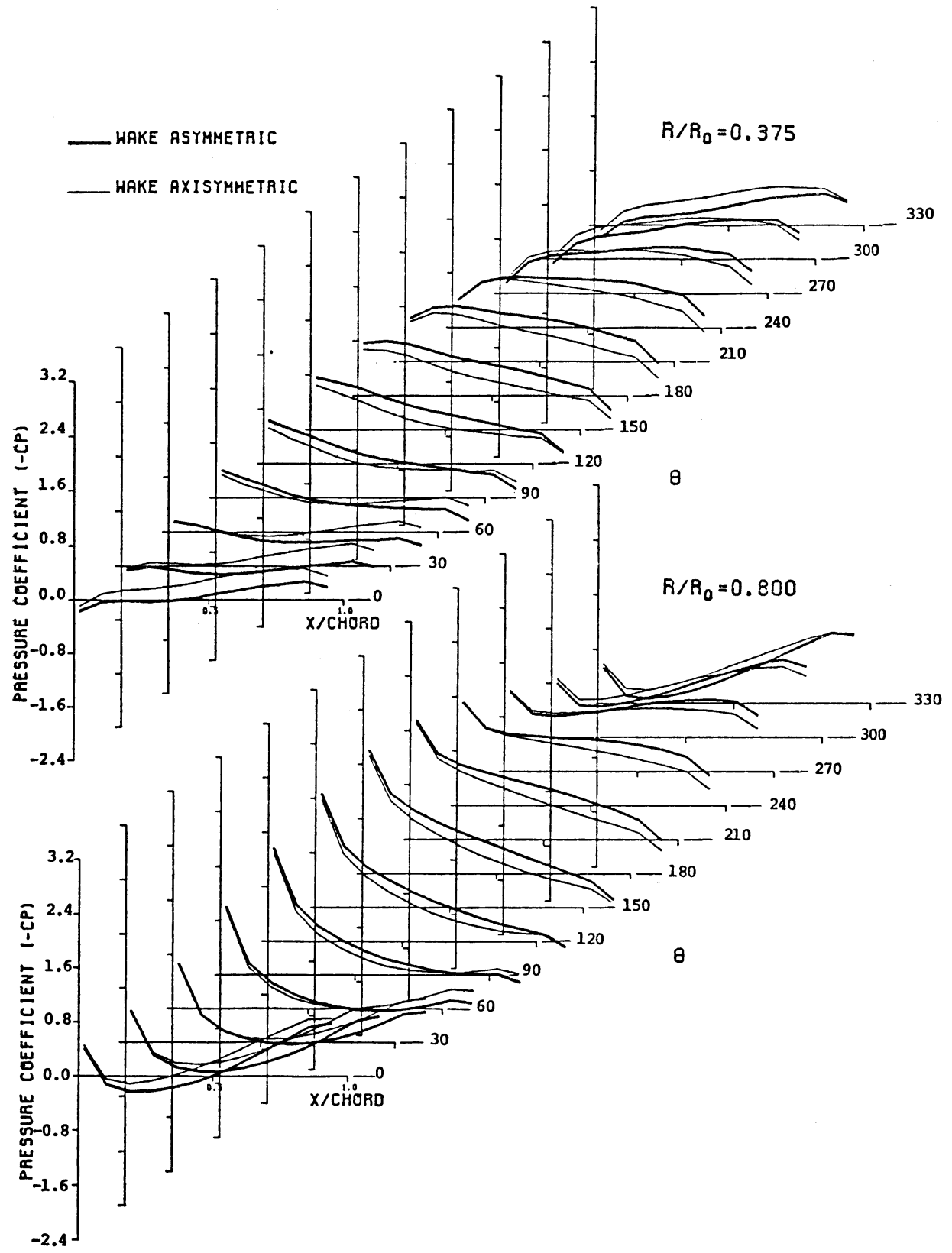

Fig. 9 Variation of pressure distribution on blade (suction side) with and without slipstream asymmetry (shaft inclination $=10^{\circ}$, without boss effects)

considering wake axisymmetry. In evaluating these results, however, it must be noted that from theoretical considerations it is unrealistic to determine cavity extent by lift equivalent method when there is such a large extent of cavity. But even in this case some general agreement has been observed with such prediction and the predicted extents have been found to be generally smaller than the observed ones. However, though there is considerable drop in pressure at around $60 \%$ chord position near the root this cannot be dealt by the present prediction method.

\section{6 Extent of erosion}

The paint peel off test was carried out in order to ascertain the extent of cavitation erosion at different cavitation numbers. All the tests were conducted for a duration of 15 minutes. Fig. 13 shows the result of such tests for $K_{T}=0.205$ 


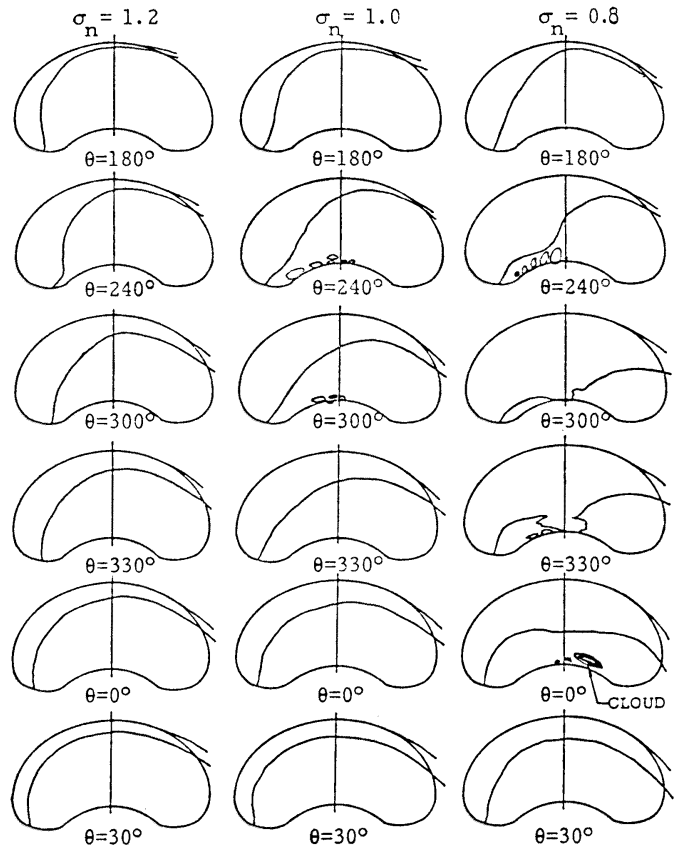

Fig. 10 Observed cavity pattern $\left(K_{T}=0.205\right.$, shaft inclination $=10^{\circ}$ ) at a shaft inclination of $10^{\circ}$ for two cavitation numbers of 1.0 and 0.8 . No erosion was observed for the cavitation number of 1.2. It can be seen that paint peel off occurs somewhere near the midchord in the root region, suggesting possible erosion in this region for continuous prolonged operation. This position of paint peel off area has been found to agree fairly well with the calculated position of drop in pressure near the root region.

\section{Conclusions}

Based on the aforementioned discussion of computer simulation and experimental results, the following conclușions had been drawn.

(1) In the pressure distribution under oblique flow conditions, between $30^{\circ}$ to $270^{\circ}$ the pressure shows the normally seen tendency of being lower at the leading edge and higher near the trailing edge. But in the range $270^{\circ} \sim 30^{\circ}$ near about $60 \%$ chord length at the root region there is a considerable drop in pressure. If the boss effects are considered this tendency is increased. This is thought to cause root cavitation as the observed position of root cavitation erosion on the blade agrees very well with this position.

(2) In case of pressure distribution by taking into account the wake asymmetry the amplitude of variation in pressure is increased and the pressure

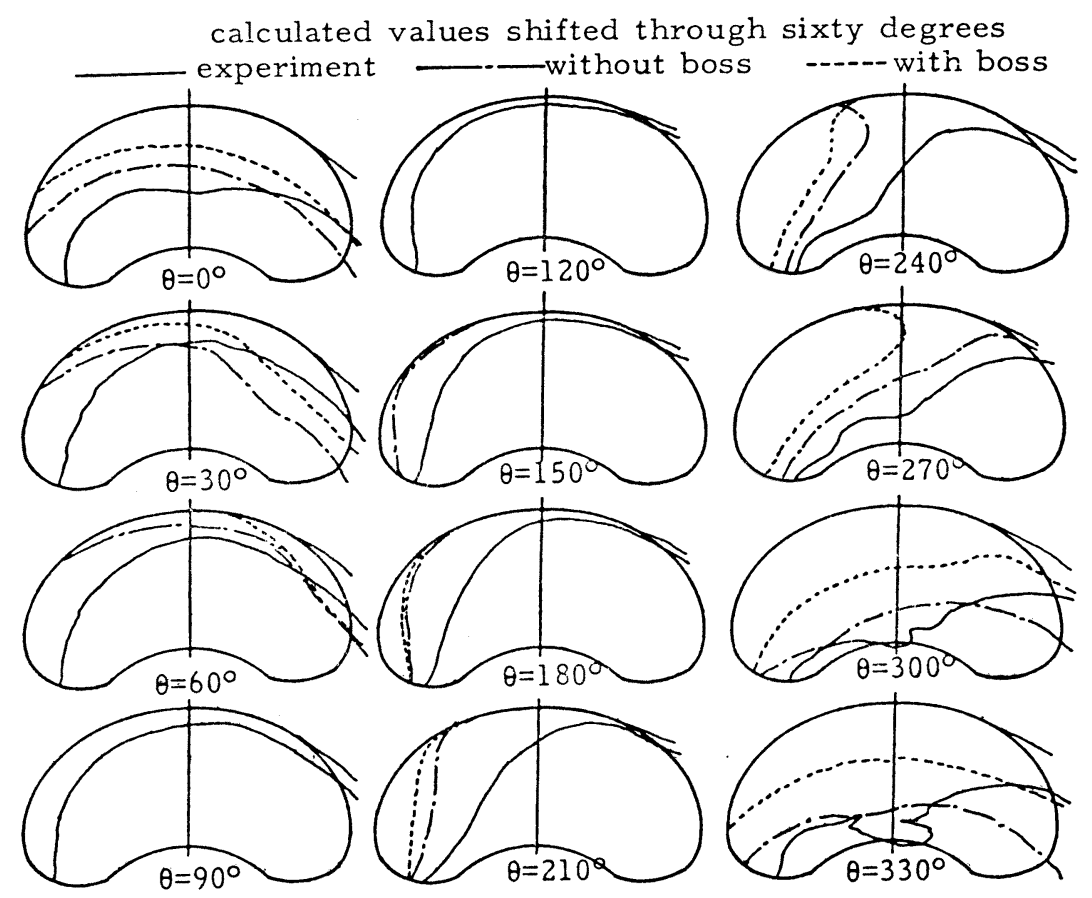

Fig. 11 Comparison of observed and calculated (with and without boss effects) cavity extents $\left(K_{T}=0.205\right.$, shaft inclination $\left.=10^{\circ}, \sigma_{\mathrm{n}}=0.8\right)$ 


\section{calculated values shifted through sixty degrees}
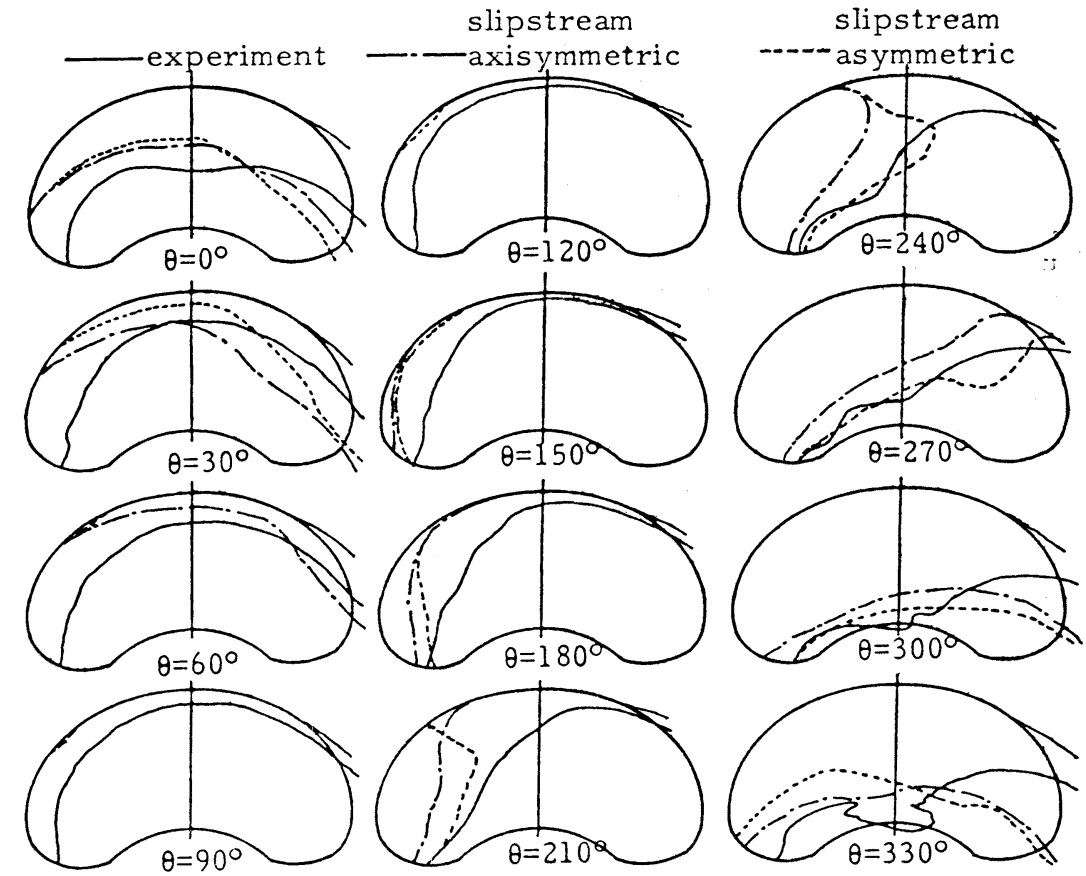

Fig. 12 Comparison of observed and calculated (with and without slipstream asymmetry) cavity extents $\left(K_{T}=0.205\right.$, shaft inclination $\left.=10^{\circ}, \sigma_{\mathrm{n}}=0.8\right)$

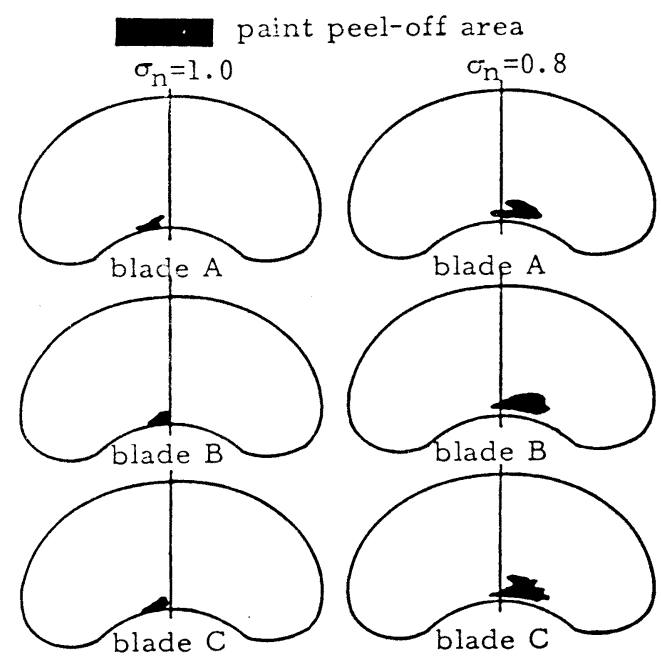

Fig. 13 Paint test results $\left(\mathrm{t}=15 \mathrm{~min} ., K_{T}=\right.$ 0.205 , shaft inclination $=10^{\circ}$ )

curve takes up a shape more susceptible to cavitation in a manner similar to that obtained by taking into account the effect of boss.

(3) The cavity extent was calculated from the computed pressure distribution using the lift eqivalent method. Since the unsteady effects of the cavity growth cannot be dealt with in the lift equivalent method, it is usual that the extent be compared with the experimentally observed ones by advancing the phase of the calculated values. In the present study if the calculated results are advanced through $60^{\circ}$ the results are found to agree well with the experimental ones.

(4) If the results of the pressure distribution are studied for different shaft inclinations it is found that at around $10^{\circ}$ or more the pressure distribution near the root takes a shape more susceptible to cavitation.

(5) By analysing the above findings of computer simulation and experiments, it can be said that the prediction of root cavitation erosion can be done with the help of the present theory for possible use in design of high speed propellers.

The calculations for the present study were per. formed on the $\mathrm{M} 280 \mathrm{H}$ system of the Computer Center, University of Tokyo. The help extended by Mr. M. Maeda, Mr. M. Miyanaga and Mr. T. Komura in conducting the experiments is gratefully acknowledged.

\section{References}

1) S. Chattopadhyay et al.: 'A study on Performance and Cavitation of Propellers for High Speed Crafts Including Effect of Boss (1 st Report- Analysis in Uniform Flow)', Journal of the Society of Naval Architects of Japan, Vol. 158 (1985). 
2) S. Chattopadhyay: 'Study of Cavitation on High Speed Propellers in Oblique Flow', $\mathrm{Ph}$. D. Thesis, Department of Naval Architecture, University of Tokyo, July (1985).

3) K. Taniguchi et al.: 'Root Erosion Experienced on the Propellers of A Destroyer', Journal of the Society of Naval Architects of Japan, Vol. 118 (1965).

4) K. Taniguchi et al.: 'Investigation into the Propeller Cavitation in Oblique Flow', Journal of the Society of Naval Architects of Japan, Vol. 121 (1967).

5) J.E. Kerwin : 'The Effect of Trailing Vortex Asymmetry on Unsteady Propeller Blade Forces', MIT, Department of Ocean Engineering Report on Contract N 00014-77-C0810, MIT OSP 85871, May (1979).

6) R. J. Boswell et al.: 'Periodic Single Blade Loads on Propellers in Tangential and Longitudinal Wakes', Propellers' 81 Symposium, SNAME, Virginia, May (1981).

7) P. G. Kozhukharov et al.: 'Investigation on Cavitating Screw Propellers in Oblique
Flow', Second International Conference on Cavitation, IMechE, Edinburgh, September (1983).

8) J. Zhi-Ye: 'Experimental Investigation on Cavitation Erosion of Propellers of $\mathrm{High}$ Speed Craft', Cavitation Erosion in Fluid Systems, The Fluids Engineering Conference, ASME, Colorado, June (1981).

9) J.E. Kerwin et al.: 'Prediction of Steady and Unsteady Marine Propeller Performance by Numerical Lifting-Surface Theory', SNAME Transactions, Vol. 86 (1978).

10) C.S. Lee : 'Prediction of the Transient Cavitation on Marine Propellers by Numerical Lifting Surface Theory', Thirteenth Symposium on Naval Hydrodynamics, Tokyo, October (1980).

11) H. Yuasa: 'Application of the Vortex Lattice Method to the Three Dimensional Theory of a Cavitating Propeller', Journal of the Society of Naval Architects of Japan, Vol. 156, November (1984). 